\title{
Mesenchymal stem cells for the treatment of neurodegenerative
}

\section{disease}

\author{
Nanette Joyce ${ }^{1}$, Geralyn Annett ${ }^{1}$, Louisa Wirthlin ${ }^{1}$, Scott Olson ${ }^{1}$, Gerhard Bauer ${ }^{1}$, and Jan \\ A Nolta ${ }^{\dagger, 1}$ \\ ${ }^{1}$ Department of Internal Medicine, Division of Hematology/Oncology, Stem Cell Program, \\ University of California, Davis, CA, USA
}

\section{Abstract}

Mesenchymal stem cells/marrow stromal cells (MSCs) present a promising tool for cell therapy, and are currently being tested in US FDA-approved clinical trials for myocardial infarction, stroke, meniscus injury, limb ischemia, graft-versus-host disease and autoimmune disorders. They have been extensively tested and proven effective in preclinical studies for these and many other disorders. There is currently a great deal of interest in the use of MSCs to treat neurodegenerative diseases, in particular for those that are fatal and difficult to treat, such as Huntington's disease and amyotrophic lateral sclerosis. Proposed regenerative approaches to neurological diseases using MSCs include cell therapies in which cells are delivered via intracerebral or intrathecal injection. Upon transplantation into the brain, MSCs promote endogenous neuronal growth, decrease apoptosis, reduce levels of free radicals, encourage synaptic connection from damaged neurons and regulate inflammation, primarily through paracrine actions. MSCs transplanted into the brain have been demonstrated to promote functional recovery by producing trophic factors that induce survival and regeneration of host neurons. Therapies will capitalize on the innate trophic support from MSCs or on augmented growth factor support, such as delivering brain-derived neurotrophic factor or glial-derived neurotrophic factor into the brain to support injured neurons, using genetically engineered MSCs as the delivery vehicles. Clinical trials for MSC injection into the CNS to treat traumatic brain injury and stroke are currently ongoing. The current data in support of applying MSC-based cellular therapies to the treatment of neurodegenerative disorders are discussed.

\section{Keywords}

amyotrophic lateral sclerosis; clinical trial; human mesenchymal stem cell; Huntington's disease; hypoxia; neurite outgrowth; neurodegenerative disease; tissue repair

Different populations of adult stem cells that can contribute to the regeneration of muscle [1,2], liver [3-6], heart [7-10] and vasculature have been described [11-13], although the mechanisms by which this is accomplished are still not completely understood. However, human mesenchymal stem cells (hMSCs) are known to secrete a variety of cytokines and growth factors that have both paracrine and autocrine activities for damaged tissues,

\footnotetext{
${ }^{\dagger}$ Author for correspondence: Stem Cell Program \& Institute for Regenerative Cures, University of California, Davis, 2921 Stockton Blvd, Room 1300, Sacramento, CA 95817, USA, Tel.: +1 916703 9308, Fax: +1 916703 9310, jan.nolta@ucdmc.ucdavis.edu.

Financial \& competing interests disclosure

The authors have no other relevant affiliations or financial involvement with any organization or entity with a financial interest in or financial conflict with the subject matter or materials discussed in the manuscript apart from those disclosed.

No writing assistance was utilized in the production of this manuscript.
} 
including the brain. The leading theory of tissue repair and regeneration by adult MSCs is that the mechanism of action is based upon the innate functions of the stem cells: the injected stem cells home to the injured area, in particular to hypoxic, apoptotic or inflamed areas, and release trophic factors that hasten endogenous repair. These secreted bio-active products can suppress the local immune system, enhance angiogenesis, reduce levels of free radicals, inhibit fibrosis and apoptosis, and stimulate recruitment, retention, proliferation and differentiation of tissue-residing stem cells. These paracrine effects are distinct from the classical model of direct differentiation of stem cells into the tissue to be regenerated.

Mesenchymal stem cells can be expanded from normal donors in large quantities and can be infused without tissue matching, since they shield themselves from the immune system [14]. Owing to the fact that MSCs present such a promising tool for cell therapy, a variety of studies initially focused not only on their characterization, but also on their utility in the treatment of several diseases in animal models. While MSCs considerably contributed to the recovery of tissues in models of myocardial infarction [15], stroke [16,17], meniscus injury [18] and limb ischemia [19], the percentage of engrafted MSCs was low in comparison with the recipient tissue cells, suggesting that their efficacy relies upon actions other than direct differentiation. Kinnaird et al. demonstrated that MSC-conditioned media stimulated endothelial cell proliferation and migration in vitro, and the injection of MSC-conditioned media into mice that had undergone hindlimb ischemia was sufficient to mediate regeneration of the blood flow in the injured limb [20]. Similar results have been demonstrated with a cardiac infarction model [21], and the secretion of multiple angiogenic cytokines from MSCs has been demonstrated. HGF, FGF-2, IGF-I and VEGF have all been detected in MSC-conditioned media. The evidence seems to point toward the theory that a complex set of trophic factors secreted by MSCs significantly contributes to injury repair in vivo, through stimulating angiogenesis, reducing oxidative stress and decreasing apoptosis.

Mesenchymal stem cells have been found to produce improvements in disease models, although a limited number of the cells could be demonstrated to be stably engrafted. A mystery that remains in the MSC field is that, in cases of acute injury or inflammation, MSCs respond to the injury robustly but only transiently, and do not become an enduring part of the repaired tissue or vasculature, to any significant degree. We have studied this in immune-deficient mice that cannot reject the human cells, so the loss of MSC appears to be independent of an immune rejection [19,22-24]. The same data have been obtained in large animal models. By contrast, when labeled MSCs are cultured in the same way, and infused intravenously into immune deficient mice that have low-level systemic damage from irradiation or a chronic disease, or no damage at all, the cells migrate through all tissues in a relatively evenly dispersed and long-lasting manner $[22,25]$. We have recovered hMSCs from numerous organs of the mice at time points from 1 to 18 months post-transplantation in those cases [22,23,25-27], whereas, in the acute injury setting, they are only transiently recovered and virtually undetectable at the area of tissue damage at 1 month postinfusion $[8,24]$. Hypoxic preconditioning of the MSCs enhanced their reparative function in a tissue ischemia model [19]. Further studies must be conducted in order to better understand the duration of the residence of infused MSCs in tissues, including the brain, and the mechanisms that recruit and retain them in chronic versus acute tissue damage. This knowledge will allow the most effective use of MSC-based cellular therapies.

\section{MSCs in neural repair: an overview}

The dogma that cells of the CNS could never regenerate has been challenged in the last decade with studies demonstrating new, migrating stem cells in the brain in many rodent injury models, as well as findings of new neurons in the adult human hippocampus [28-31]. There is currently a great deal of interest in the use of MSCs to treat neurodegenerative 
diseases, in particular those that are fatal and difficult to treat, through providing neurotrophic factors to encourage repair and, potentially, new growth of neurons. Proposed regenerative approaches include delivery via intracerebral or intrathecal injection, or even infusion via an intranasal route [28]. Therapies will capitalize on innate trophic support from MSCs or from the delivery of augmented growth factors, such as brain-derived neurotrophic factor (BDNF) or glial-derived neurotrophic factor (GDNF), into the brain to support injured neurons, using genetically engineered MSCs as the delivery vehicles.

Upon transplantation, MSCs in the brain promote endogenous neuronal growth, decrease apoptosis and regulate inflammation, primarily through the use of secreted factors. MSCs can mediate modification of the damaged tissue microenvironment to enhance endogenous neural regeneration and protection. MSCs transplanted at sites of nerve injury have been demonstrated to promote functional recovery by producing trophic factors that induce survival and regeneration of host neurons [29]. Transplantation of human bone marrow stem cells into the brain of immunodeficient mice markedly increased the proliferation of endogenous neural stem cells [30]. In an experimental allergic encephalomyelitis model of multiple sclerosis (MS), rodents that received an intraventricular infusion of MSCs were found to have almost twice the number of axons as control animals [31]. Although candidate molecules are under investigation, further detailed studies are needed to define the factors responsible for the MSC-mediated induction of proliferation in resident neural stem cells, in order to best capitalize upon this type of therapy for the repair of neurodegenerative diseases.

Numerous clinical trials have demonstrated the biosafety of systemic infusion of allogeneic MSCs into patients with various diseases. Monitoring of biosafety aspects has been performed throughout these trials. For direct infusion of MSCs into the brain or spinal cord, the biosafety aspects will be monitored extremely carefully. Risks could include development of cytogenetic abnormalities in cultured cells or ectopic differentiation to other tissue lineages. The development of cytogenetic abnormalities when MSCs are cultured for a long period of time, past the crisis point, can be observed in rodent MSCs, which do not reflect hMSC biology well and are almost always riddled with phagocytic monocyte/ macrophage elements [23]. There has been one report of a karyotypic abnormality occuring in hMSCs that were cultured in conditions that would never be allowed in a good manufacturing practice or good laboratory practice setting [32]. When hMSCs are cultured under good laboratory practice/good manufacturing practice conditions, adverse events have not been observed. We have published a decade-long biosafety study on this aspect of their clinical safety profile [26].

However, a potential risk for infusion of MSCs into the spinal cord or brain is that they have potent revascularization capacity and will home to the hypoxic region around a tumor bed $[33,34]$. This tropism for the tumor or wound bed could result in enhanced revascularization and survival of a growing tumor. Therefore, in proposed cellular therapy trials an important exclusion criteria is the lack of prior brain tumors or other cancers in the past 5 years. A pretreatment MRI, which would be carried out in many neurodegenerative disease trials to establish baseline, could also be used to rule out existing brain tumors.

Several groups have reported that MSCs can be induced to express neural markers in vitro, in particular after cocultivation with human or murine neurons or conditioned medium. However, although mature neurons with functional activity can be generated in culture from neural stem cells $[35,36]$, it has not been demonstrated that mature neurons with signaling capacity can be generated from MSCs; thus calling into question the authenticity of this transdifferentiation and the ability of MSCs to produce authentic neural derivatives [37]. MSCs can assimilate proteins and pieces of membrane from other cells. Thus, more 
definitive proof must be found for transendocytosis, trogocytosis, exosomal transport, shuttle through nanotubules or cell-contact-dependent intercellular transfer to be ruled out. Cellular communication through the intercellular exchange of intact membrane patches, also called trogocytosis, is a ubiquitous phenomenon first described in cells of the immune system [38]; but, through the use of advanced videomicroscopy techniques, has also been observed in MSCs [O $\mathrm{OLSON}_{\mathrm{S}} \mathrm{S}, \mathrm{Nolta}_{\mathrm{J}} \mathrm{JA}, \mathrm{U}_{\text {NPUBLISHed }} \mathrm{D}_{\text {ATA }}$ ]. Transfer of organelles as large as mitochondria from MSCs to damaged cells has also been described [39]. Therefore, reports of MSC transdifferentiating to neural phenotypes, particularly after cocultivation with neurons, must be cautiously interpreted. However, the ability of MSCs to migrate to areas of damage in the brain and to secrete beneficial neurorestorative factors (Figure 1) is significant and is the primary focus of this article.

\section{Expression of neuroregulatory factors by MSCs}

Understanding the innate capacity of MSCs to influence neural cell growth, survival and neurite extension is currently a very important field of study. Detailed determination of which factors MSCs express, and under which conditions, is key. Crigler et al. demonstrated that specific subpopulations of hMSCs expressed BDNF and $\beta$-NGF but not neurotrophin-3 and -4 . They used a co-culture assay to demonstrate that BDNF expression levels correlated with the ability of MSC subclones to induce survival and neurite outgrowth in the SH-SY5Y neuroblastoma cell line. The effects were only partially inhibited by a neutralizing antiBDNF antibody, indicating that other factors secreted by the MSCs also had neuroregulatory effects. The authors identified production of other neurite-inducing factors, axon guidance and neural cell adhesion molecules, which contributed to the capacity of the MSCs to induce neuronal cell survival and nerve regeneration [29]. Wilkins et al. have also demonstrated that MSCs secrete BDNF and that addition of anti-BDNF neutralizing antibodies attenuated their neuroprotective effects. These studies help to, at least partially, determine the mechanisms of neuroprotection mediated by MSCs, and also validate the important role for BDNF in neuronal protection [40].

The Wnt antagonist Dickkopf (Dkk)-1 is another candidate for MSC-mediated neuritogenesis. Endo et al. demonstrated that Dkk-1 promotes neurite outgrowth in Ewing sarcoma family tumor cells, which were identified as neurites. This process appeared to occur by causing endogenous Wnts to switch from stimulation of the $\beta$-catenin pathway to noncanonical signaling [41]. Etheridge $e$ al. identified Dkk-1 as a product of MSCs [42], and Gregory and coworkers demonstrated that Dkk-1 expression by MSCs is cell-density dependent [43]. Further study of the role of Dkk-1 in MSC-mediated neuritogenesis is warranted. A deeper understanding of the processes involved in axonal pathfinding is crucial if the system is to be manipulated to promote nervous system repair [44].

In addition to neurotrophic factors, the extracellular matrix molecules produced by MSCs have also been demonstrated to support neural cell attachment, growth and axonal extension [45]. Neurons on extracellular matrix derived from MSCs formed more complex and extended neurite networks than those cultured on poly-D-lysine [45]. Croft et al. demonstrated that soluble factors produced by MSCs promoted the survival of neurons in culture and promoted axonal growth in neuronal progeny [46]. They proposed mechanisms to manipulate MSCs in vitro, prior to transplantation, which could potentially further enhance the endogenous neurogenic response to injury [46].

Mesenchymal stem cells also provide an excellent platform from which to produce additional factors for neural regulation, which can be introduced using viral vectors under inducible promoters, for the treatment of specific diseases. Foremost among these are BDNF 
and GDNF. Discussion of these approaches in the context of treatments under consideration for a selection of neurodegenerative diseases are presented later.

\section{Huntington's disease}

Huntington's disease (HD) is a currently incurable inherited genetic disorder for which the condition of the patients inexorably worsens and their prognosis is, unfortunately, eventual death, following decline in emotion, movement and, finally, cognition. The pathology of HD is caused by a variably sized polyglutamine expansion of the protein product of the huntingtin $(h t t)$ gene. Multiple disease mechanisms have been elucidated for HD and are currently under investigation in the search for therapeutics. Currenly, the best hope for halting HD progression is to reduce or eliminate the mutant htt protein in the affected cells [47,48]. siRNAs have been demonstrated to be effective at reducing htt levels and ameliorating disease symptoms in animal models $[49,50]$. Exciting new data show that the mutant $h t t$ mRNA can be specifically targeted, while sparing the transcript produced by the normal allele [51-53]. The challenge for this technology is to deliver the siRNA into the human brain in a sustained, safe and effective manner. HD is a challenging disease to treat. Not only do the affected, dying neurons need to be salvaged or replaced, but also the levels of the toxic mutant protein must be diminished to prevent further neural damage and to halt progression of the movement disorders, and the physical and mental decline that is associated with HD.

Lescaudron and colleagues transplanted autologous bone marrow stem cells in the damaged striatum of a rat HD model and reported that the transplant significantly reduced working memory deficits [54]. The transplanted cells remained undifferentiated but exerted trophic effects. Bantubungi et al. reported that the lesioned environment of the striatum favored the proliferation and intralesional distribution of MSCs [55]. Several groups have demonstrated that striatal atrophy in models of HD is accompanied by the subsequent enlargement of lateral ventricles [56,57]. The reduction in volume of the lateral ventricle can, thus, be a good indicator of repair in HD correction studies. Amin et al. demonstrated that, following MSC implantation into the striata of rats with a unilateral damage model of HD, striatal atrophies were significantly reduced and, consequently, the volume of the lateral ventricle returned to a significantly smaller size, approaching the normal contralateral ventricle [56]. Their results demonstrated the potential for MSCs in the treatment of microanatomical defects in the motor disorders of HD.

Data suggest that MSC implantation into the striatum could potentially delay the inexorable loss of medium spiny neurons in HD. An important factor when considering the use of MSCs to help augment recovery and survival of striatal neurons for HD patients is that the MSCs implanted at adjacent sites should have the capacity to migrate into the most damaged areas in response to stress or death of medium spiny neurons, which are lost in HD. MSCs are known to migrate robustly, in response to injury in the brain. Delcroix et al. demonstrated that iron nanoparticle-labeled MSCs migrated from an implantation site in the subventricular zone microenvironment towards the olfactory bulb through the rostral migratory stream, only when a mechanical lesion of the olfactory bulb was performed [58]. They confirmed the known potential of iron-labeled MSCs to migrate towards areas of damage, even over a great distance in the brain. The migratory capacity of MSCs was further examined by transplantation of the cells at a distance from a quinolinic acid-induced striatal lesion, a rat model for HD. Migration was monitored using MRI scans followed by histology. The engrafted MSCs were demonstrated to have migrated a great distance along the internal capsule toward the quinolinic acid-induced lesion in the striatum. They were demonstrated to have regenerated the damaged striatal dopaminergic nerve terminal network in this animal model for HD [59,60]. 
A human cellular therapy trial has already demonstrated progress in the treatment of HD by intracerebral implantation. Bachoud-Lévi and colleagues transplanted human fetal neural stem cells into the brains of five patients with HD $[61,62]$. Three out of the five patients with HD showed motor and cognitive improvements 2 years after the fetal neural graft. Clinical improvement plateaued after 2 years and then showed variable decline 4-6 years after the implantation $[61,62]$. These and other studies demonstrate that cells can be safely implanted into the brain without adverse events. However, a major challenge yet to be robustly overcome is to induce the new neurons to make bona fide synaptic connections. Cotransplantation of fetal or embryonic stem cell-derived neural grafts with MSCs to enhance neuritogenesis and to guide axonal pathfinding through areas of damage could be considered. Another confounding factor for the treatment of HD is that the brain microenvironment will be continually damaged owing to the presence of the mutant $h t t$ RNA and protein, even if new, healthy neurons are implanted. Efforts to specifically knockdown the expression of this mutant $h t t$ RNA and protein prior to or simultaneously with the MSC-based therapies must be considered [51-53]. BDNF therapy is a leading candidate for use in HD since it has been linked mechanistically with the underlying genetic defect. Striatal neurons depend on BDNF levels for function and survival [63]. In the later stages of HD, the levels of available BDNF plummet since the mutant htt protein and associated factors interfere with normal trafficking of the protein $[64,65]$. This reduction of BDNF levels affects the onset and severity of the disease in HD mouse models [63]. Upregulation of BDNF in the forebrain of R6/1 mice (a model of HD) improves the disease symptomatology [66]. Additional transgenic rodent models of HD have also demonstrated amelioration of the disease phenotype by enforced exogenous expression of BDNF (reviewed in [67]). Thus, owing to its prosurvival effects in striatal neuropathology, BDNF is the main candidate for neuroprotective therapies (reviewed in [68]).

Mesenchymal stem cell transplantation may help stabilize the striatal environment by producing anti-inflammatory cytokines and neurotrophic factors, including BDNF, among others, that they have been demonstrated to be produced in the brain. Dey et al. demonstrated that MSCs, especially those engineered to over-express BDNF, had significant ameliorative effects on disease progression in a mouse model of HD [69]. The use of MSCs to deliver factors, both through their own innate responses and through engineering, has benefits over direct protein administration, because transplanted MSCs can provide sustained and long-term delivery of factors at supraphysiological levels, as we and others have demonstrated over the past two decades [22,23,27,70-73].

Efforts from our laboratory and others are currently evaluating the effects of BDNF expression from hMSCs implanted into the striata of immunocompromised HD mice. Using immune-deficient mouse models, we have recovered hMSCs that had maintained expression of the gene product from numerous organs, including the brain, at time points from 1 to 18 months post-transplantation [22,25,27,70,72,74,75]. We also performed a 10-year-long biosafety study to demonstrate that genetically engineered hMSCs are safe and do not cause adverse events in vivo [26]. The need for safe and effective cellular therapies to treat HD is great, since current therapies only target symptoms, and there are currently no drugs or other treatments that effectively delay the inexorable loss of striatal volume in affected patients.

\section{Amyotrophic lateral sclerosis}

Amyotrophic lateral sclerosis (ALS) is a relentless neurodegenerative disease caused by the selective destruction of motor neurons in the motor cortex, brainstem and spinal cord. The steady progressive loss of motor neurons throughout the neuraxis causes muscle atrophy, weakness and immobility. Premature death, typically within 5 years of diagnosis, is inevitable and is most often caused by paralysis of respiratory muscles with subsequent 
respiratory failure. Currently ALS is incurable; treatment consists of therapies aimed at symptomatic relief and Rilutek ${ }^{\circledR}$ - the only US FDA-approved disease-modifying drug for ALS - provides modest life-prolonging benefit. Development of relevant therapies has been challenging and elusive, complicated by the lack of understanding of the underlying inciting pathophysiology.

Amyotrophic lateral sclerosis is largely a sporadic disease with an unclear cause. However, approximately $5-10 \%$ of patients diagnosed with ALS have an inherited, familial ALS form of the disease, which shares nearly identical clinical and histopathologic hallmarks with sporadic ALS [76]. While many disease-causing mutations have been identified, the most common are point mutations within the gene encoding for $\mathrm{Cu} / \mathrm{Zn}$ superoxide dismutase (SOD)1. This clear genetic link has led to the development of transgenic rodent models carrying various mutant human SOD1 genes (i.e., point mutations with amino acid substitutions G37R, G85R and G93A), which cause clinical manifestations, mimicking both sporadic ALS and familial ALS. Since their development, better understanding of disease pathophysiology has ensued with mounting evidence to support the concept of a multifactorial disease process culminating in apoptosis of motor neurons. Mechanisms implicated in this process include: glutamate excitotoxicity, oxidative damage, cytoskeletal abnormalities, endoplasmic reticulum stress from abnormal cellular protein products, mitochondrial dysfunction, abnormal microglial and astrocyte function, and impaired neurotrophic support [77-81].

Stem cell therapies hold significant promise for clinical benefit by offering both the possibility of cellular replacement, as well as targeted gene modification and neurotrophic factor delivery to interrupt these abnormal mechanisms. Based on their unique properties, MSCs are playing a key role in developing treatment strategies. Both murine MSCs and hMSCs have been delivered with varying techniques to transgenic SOD1 ALS rodent models to evaluate safety, effectiveness and disease-altering properties. Zhao and colleagues performed intravenous injection of hMSCs into presymptomatic irradiated G93A mice [82]. They reported that the hMSCs survived over 20 weeks in the recipient mice, integrated into the parenchyma of both the brain and spinal cord. The transplanted mice had both delayed onset and slower disease progression with an increased lifespan when compared with the untreated mice [82].

Several groups have demonstrated that intraparenchymal delivery of hMSCs is safe and can delay loss of motor neurons in rodents. Vercelli et al. transplanted hMSCs directly into the lumbar spinal cords of transgenic SOD1 mice. The MSCs migrated throughout the spinal cord and delayed loss of motor neurons, prolonging motor performance [83]. Another study compared the efficacy of transplanting olfactory ensheathing cells and rat MSCs intrathecally through the fourth ventricle in the spinal cord. Although the olfactory ensheathing cells distributed widely, no significant changes in clinical outcomes were observed until after MSC transplantation, when female ALS mice showed statistically longer disease duration than males and control mice [84].

Quantitative pathological analysis has been carried out to examine the neuromuscular junctions, ventral root and spinal cord at multiple ages in the G93A mouse model. Fischer et al. reported histopathologic abnormalities of the neuromuscular junctions as the first sign of disease onset [85]. Approximately $60 \%$ of the ventral roots suffered damage, with decline of the neuromuscular junctions, prior to any development of abnormalities in the motor neuron cell body or neuroglia [85]. GDNF is a promising factor in that it has a high affinity for motor neurons and can prevent their death. The protein is large and does not cross the blood-brain barrier, so it is difficult to directly administer to the brain. Svendsen's group demonstrated that human neural progenitors isolated from the cortex and modified to secrete 
GDNF survived up to 11 weeks in the lumbar spinal cord of rats overexpressing the G93A SOD1 mutation. Cellular integration into both gray and white matter was observed with secretion of GDNF within the region of cell survival. Fibers upregulated cholinergic markers in response to GDNF, indicating that it was physiologically and locally active [86]. Central implantation of GDNF-secreting neural precursor cells by the same group improved maintenance of spinal motor neurons but failed to improve hindlimb function [87]. Suzuki et al. delivered intramuscular hMSCs that secreted GDNF [88]. The MSCs survived within the muscle, provided continuous neurotrophic support, increased the number of neuromuscular junctions, ameliorated loss of motor neurons within the spinal cord, and improved survival and function in ALS rats [88]. Taken together, these intriguing results provide support for the role of multitargeted treatment strategies and the potential for MSCs to deliver augmented neurotrophic support.

Many trophic factors have been studied using adeno-associated viral (AAV)-mediated delivery in ALS. AAV GDNF, IGF-1 and VEGF have demonstrated promising effects in rodent models, by increasing axonal outgrowth, blocking neuronal apoptosis and promoting neurogenesis. AAV-delivered HGF retards the progression of disease in the transgenic SOD1 mouse model. In addition to direct neurotrophic activities, HGF functions on the astrocytes of G93A mice to maintain levels of EAAT2, a glial-specific glutamate transporter that might be responsible for the reduction of glutamatergic neurotoxicity of motor neurons. In addition, HGF is capable of reducing astrocytosis and microglial accumulation [89,90]. However, AAV-mediated delivery presents clinical challenges for treatment in humans and there are some safety concerns [91].

Neurotrophic factors that provided benefit in the murine model have had mixed results in humans. A total of three Phase III clinical trials using IGF-1 protein have failed to produce consistent meaningful effects when delivered systemically through subcutaneous injection. After intrathecal IGF-1 delivery showed promise in the SOD1 G93A mice [92], Nagano et al. completed a small double-blind clinical trial to assess the effect of intrathecal administration of IGF-1 on disease progression in nine patients with ALS [93]. They received either high-dose ( $3 \mu \mathrm{g} / \mathrm{kg}$ of bodyweight) or low-dose $(0.5 \mu \mathrm{g} / \mathrm{kg}$ of bodyweight $)$ IGF-1 every 2 weeks for 40 weeks. The high-dose treatment slowed the decline of motor functions, but not bulbar function or vital capacity [93]. This may be caused by gravitational effects on the medication and the spinal level of intrathecal delivery. MSCs that have been genetically engineered to produce IGF-1 may provide superior efficacy, as it is well established that MSCs migrate through the parenchyma, homing to areas of cellular distress, providing the opportunity for discrete targeted delivery. Efforts from our laboratory are currently exploring the effects of IGF-1 expression from hMSCs implanted into both the peripheral muscle and spinal cord of SOD1 G93A mice [Joycen etal., $\mathrm{U}_{\text {Npublished }} \mathrm{D}_{\text {ata }}$ ].

A human cellular therapy trial has already demonstrated progress in the treatment of ALS by intraspinal injection. After characterization of bone marrow-derived MSCs, Mazzini and colleagues transplanted the autologous MSCs into the thoracic spinal cord of nine patients with ALS [94]. No significant acute or late side effects were reported and four of the patients showed significant slowing of the linear decline of forced vital capacity and the ALS-Functional Rating Scale score [94]. A Phase II clinical trial using MSCs is underway in Europe, and the FDA has recently approved a Phase I trial in the USA. The need for safe and effective cellular treatments is great in ALS. These therapies offer hope to patients and their families struggling with this devastating disease. 


\section{Parkinson's disease}

The goal of cellular therapy to treat Parkinson's disease (PD) is the replacement of lost neurons in the substantia nigra with healthy dopaminergic neurons or the protection of these neurons from further loss. Potential sources for cell replacement have included fetal ventral mesencephalon tissue and various stem cell types that can differentiate into dopaminergic neurons. Numerous experimental replacement therapies have been examined in preclinical animal models and in clinical trials (reviewed in $[95,96]$ ). MSCs have also been examined in animal models of PD, for their neurorestorative effects and as delivery vehicles for production of additional factors.

Jin et al. demonstrated that MSC-mediated secretion of neurotrophic factors, such as NGF and BDNF, could upregulate tyrosine hydroxylase gene expression in rat E13.5 ventral mesencephalic cells [97]. The ventral mesencephalic cell groups that had been co-cultured with MSCs showed a higher expression of both tyrosine hydroxylase and dopamine than those that had not been co-cultured with MSCs. These proteins were not expressed in the MSCs, indicating that the co-culture had upregulated expression in the neuronal cells [97]. Bouchez et al. studied the effects of transplantation of bone marrow-derived MSCs in a rat model of PD. The MSC graft was demonstrated to reduce the behavioral effects induced by a 6-hydroxydopamine lesion (a model for PD), and was demonstrated to partially restore the dopaminergic markers and vesicular striatal pool of dopamine [98]. Sadan et al. conducted an efficacy study using the 6-hydroxydopamine-induced lesioned rat model of PD. MSCs or BDNF-secreting MSCs were transplanted on the day of 6-hydroxydopamine administration. Amphetamine-induced rotations were measured as a primary behavior index. An MRI study conducted with iron-labeled cells, followed by histological verification, revealed that the engrafted cells migrated toward the 6-hydroxydopamine lesion, and regeneration of the damaged striatal dopaminergic nerve terminal network was observed. The BDNF-modified MSCs ameliorated amphetamine-induced rotations by $45 \%$ and inhibited dopamine depletion. In these studies, the neurotrophic factor-modified MSCs were more effective than the innate, unmodified MSCs [99].

Glial-derived neurotrophic factor and its relative, neurturin, have been demonstrated to restore function in damaged dopaminergic neurons (reviewed in [100]). A major barrier to clinical translation has been delivery. Factors delivered by intracerebral injection in patients have been ineffective, probably owing to limited distribution within the putamen. A recent trial of neurturin therapy showed promise but, unfortunately, did not achieve significance in the Phase II evaluation [101]. The trial had used an AAV vector to deliver neurturin, a technique that had been demonstrated to be effective in preclinical animal trials (reviewed in [102]). However, the human brain is vast and the vector probably did not achieve sufficiently widespread distribution to allow correction of significant regions of the damaged neurons. By contrast, delivering MSCs near the damaged region of the brain might be an option to deliver neurturin more widely, since they would continue to migrate to the most damaged parts of the brain, using natural chemotaxis and will pump out their transgene product for at least 18 months. Therefore, the use of MSCs as the delivery vehicle, rather than vector alone, could potentially have the capacity to improve and extend the results observed in the prior neurturin trial.

In a study recently reported by $\mathrm{Li}$ et al. two subjects with $\mathrm{PD}$, who had transplanted fetal mesencephalic dopaminergic neurons that had survived for over 10 years, developed $\alpha$ synuclein-positive Lewy bodies in the engrafted donor neurons [103]. Although the majority of grafted cells were functionally unimpaired after a decade and the recipients still experienced long-term symptomatic relief, this study provided evidence that the disease can propagate from host to graft cells. MSCs are highly interactive with their microenvironment, 
and can share protein, RNA and even mitochondria with damaged tissues [OLSONSETAL,

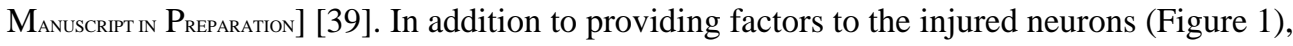
MSCs could also be susceptible to accruing material from the damaged host cells in PD, Alzheimer's disease and HD. This is an interesting aspect to clinical MSC therapies and should be monitored in preclinical studies.

\section{Clinical trials of MSCs for neural repair}

The ability of MSCs to secrete factors to decrease motor neuron death when implanted into the CNS of ALS patients was assessed in a human clinical trial conducted by Mazzini et al. [94,104]. Ten patients with ALS who had severe functional impairment of their legs were enrolled in the MSC clinical trial with no adverse events. MSCs were isolated from the patients' own bone marrow and expanded using good manufacturing practice conditions. Expanded MSCs were suspended in the autologous cerebrospinal fluid and transplanted into the recipient's spinal cord at a high thoracic level. No immediate or delayed transplantrelated toxicities were observed. Patients were enrolled and regularly monitored before and after transplantation using clinical, psychological, neuroradiological and neurophysiological evaluations. At 3 months after cell implantation, a trend toward slowing of the decline in muscular strength was observed in the legs of four of the first seven patients treated. Since this was not a randomized study, the results are not definitive; however, they do show that MSC infusion into the cerebrospinal fluid could be tolerated without adverse events in patients with ALS [94].

A pilot trial using either intravenously or intrathecally injected MSCs has been conducted by Karussis and colleagues in 12 patients diagnosed with untreatable neurodegenerative diseases - ALS or MS [105]. Their intent was to demonstrate the safety of the delivery methods and treatment with autologous MSCs. Karussis et al. initiated the pilot trial after in vitro and preclinical investigations had demonstrated immunomodulatory effects of MSCs with suppression of self-reactive T lymphocytes [201]. In addition, animal studies in mice with chronic progressive experimental autoimmune encephalomyelitis showed mitigation of their clinical course accompanied by histopathologic evidence of neuroregeneration following intracranial and intravenous injection of MSCs. One adverse event was reported during the course of the pilot study - one patient experienced transient meningeal irritation following the intrathecal delivery route. The authors speculated that the symptoms were caused by exposure of residual dimethyl sulfoxide after improper resuspension of frozen cells by an inexperienced technician [105]. This study was extended to include ten ALS and ten MS patients, demonstrating proof of concept that both delivery methods were feasible and safe. The group are currently conducting a larger Phase I/II trial delivering autologous MSCs as a treatment in patients with severe refractory MS [201].

Multiple sclerosis is generally considered an autoimmune disease, characterized by chronic progressive CNS demyelination and inflammation, leading to severe disability. Many Phase I/II trials have been conducted in the MS patient population using hematopoietic stem cell transplantation, with reports of prolonged symptom-free periods and reduced functional impairment [106-111]. However, conditioning regimens, including lymphocytic ablation, have significant risks of increased morbidity and mortality $[107,110]$. Case reports and a small pilot study including ten patients with MS treated with autologous MSCs have been conducted with intriguing results and few, if any, adverse events $[112,113]$. Treatment with autologous MSCs, based on their immunomodulatory and neuroregenerative properties, is now being investigated as a safer alternative to hematopoietic stem cell transplantation [107]. Currently, there are at least four clinical trials being conducted at centers in England, Israel, Spain and the USA, which are evaluating the safety and efficacy of bone marrowderived MSCs in the treatment of MS [202]. 
Direct injection of MSCs into the injured region of the brain during surgery following traumatic brain injury has also been performed without adverse events. Seven traumatic brain injury patients each received up to $10^{-9}$ expanded MSCs during the cranial repair operation [114]. Patients were followed up for 6 months and demonstrated significant improvements in neurologic function. Placebo-controlled trials for MSC injection into the CNS for traumatic brain injury and stroke, as well as spinal cord injury and neurodegenerative disorders are currently ongoing in countries outside of the USA [203]. No adverse events have been reported from these studies. Clinical trials of an MSC-like multipotent cellular product, MultiStem ${ }^{\circledR}$, has been approved by the FDA to treat stroke in the USA $[115,116]$. The clinical trial is currently being conducted by the company Athersys (OH, USA). Osiris therapeutics (MD, USA) has conducted multiple clinical trials using allogeneic MSCs administered through systemic infusion. No stem cell-related events have occurred and their studies provide extensive safety and provisional efficacy data for allogeneic bone marrow-derived MSC administration to patients through FDA-approved clinical trials [117-119].

The potential benefit-to-risk ratio and, in particular, safety are always the foremost considerations by the FDA. This article has covered the potential benefits of MSC-based therapies for the treatment of neurodegenerative diseases. We and many others have documented the biosafety of MSC therapies, which are now in Phase III trials for some indications, and further safety and efficacy data are being collected by numerous groups. Extending MSC-based therapies to neurodegenerative diseases, in particular those for which there are currently no effective treatments, such as HD and ALS, would have a high potential benefit-to-risk ratio.

\section{Future perspective}

This article reviews several strategies for MSC-based neural repair that are under consideration or are currently being tested in human Phase I clinical trials. We predict that the future of this area of regenerative medicine will encompass even more applications for this approach. Adequate biosafety and efficacy studies have now been completed in multiple animal models to allow safe Phase 1 clinical trials following all approvals by regulatory agencies.

A biosafety concern remains when considering the use of genetically engineered MSCs. The random integration of vectors carrying genes for neurotrophic or other factors could run the risk of insertional integration. However, the fields of homologous recombination and targeted gene delivery are rapidly advancing and we predict that integration into a 'safe harbor' site in MSCs could be carried out in the near future.

Another exciting advance is the generation of MSCs from human embryonic stem cell and induced pluripotent stem cell lines. Since human embryonic stem cell and induced pluripotent stem cell lines are continually dividing, clonally derived lines with genes that have been successfully integrated into a safe site, they could be continually expanded and fully characterized prior to differentiation to MSCs. Whether these human embryonic stem cell and induced pluripotent stem cell-derived MSCs will have the same functions in vivo and similar safety profiles as the adult MSCs remains to be determined.

In summary, we predict that in future years, infusion of banked, genetically engineered allogeneic or cell line-derived MSCs will be used to treat multiple areas of human neural disease and damage, including additional neurodegenerative diseases and acute brain injuries. 


\section{Executive summary}

\section{Mesenchymal stem cells in neural repair: an overview}

- Upon transplantation, mesenchymal stem cells (MSCs) in the brain promote endogenous neuronal growth, decrease apoptosis and regulate inflammation, primarily through the use of secreted factors.

- Numerous clinical trials have demonstrated the biosafety of systemic infusion of allogeneic MSCs into patients with various diseases. Monitoring of biosafety aspects has been performed throughout these trials.

- Therapies will capitalize upon the innate trophic support from MSC or on augmented growth factor support.

\section{Expression of neuroregulatory factors by MSCs}

- MSCs can secrete multiple factors that stimulate growth of neural progenitors and neuritogenesis.

- In addition to neurotrophic factors, the extracellular matrix molecules produced by MSCs have also been demonstrated to support neural cell attachment, growth and axonal extension.

- MSCs also provide an excellent platform from which to produce additional factors for neural regulation, introduced using viral vectors under inducible promoters for the treatment of specific diseases.

\section{Disease models}

- The transplantation of MSCs directly into the brain has been demonstrated in models of Huntington's, Parkinson's and other diseases.

\section{Clinical trials of MSCs for neural repair}

- Ongoing clinical trials of MSC infusion into the brain and spinal cord (tissues of the CNS) are discussed.

- Extending MSC-based therapies to neurodegenerative diseases, in particular those for which there are currently no effective treatments, such as Huntington's disease and amyotrophic lateral sclerosis, would have a high potential benefitto-risk ratio.

\section{Acknowledgments}

The authors contributed as follows: N Joyce, G Annett and JA Nolta: conception and design, collection and/or assembly of data, data analysis and interpretation, manuscript writing and final approval of the manuscript. S Olson and L Wirthlin: collection and/or assembly of data, manuscript writing and final approval of the manuscript. G Bauer: manuscript writing and final approval of the manuscript.

This work was funded by the California Institute for Regenerative Medicine (CIRM) grant number TR1-01257 (JA Nolta), CIRM Training Grant to UC Davis (N Joyce), NIH NHLBI 1RO1 HL073256-01 (JA Nolta) and 5P30AG010129-19 (DeCarli PI: JA Nolta and S Olson - pilot project PIs).

\section{Bibliography}

Papers of special note have been highlighted as:

- of interest 
-. of considerable interest

1. Williams JT, Southerland SS, Souza J, et al. Cells isolated from adult human skeletal muscle capable of differentiating into multiple mesodermal phenotypes. Am Surg 1999;65:22-26. [PubMed: 9915526]

2. Galmiche MC, Koteliansky VE, Briere J, et al. Stromal cells from human long-term marrow cultures are mesenchymal cells that differentiate following a vascular smooth muscle differentiation pathway. Blood 1993;82:66-76. [PubMed: 8324235]

3. Theise ND, Nimmakayalu M, Gardner R, et al. Liver from bone marrow in humans. Hepatology 2000;32:11-16. [PubMed: 10869283]

4. Theise ND, Badve S, Saxena R, et al. Derivation of hepatocytes from bone marrow cells in mice after radiation-induced myeloablation. Hepatology 2000;31:235-240. [PubMed: 10613752]

5. Petersen BE, Bowen WC, Patrene KD, et al. Bone marrow as a potential source of hepatic oval cells. Science 1999;284:1168-1170. [PubMed: 10325227]

6. Lagasse E, Connors H, Al-Dhalimy M, et al. Purified hematopoietic stem cells can differentiate into hepatocytes in vivo. Nat Med 2000;6:1229-1234. [PubMed: 11062533]

7. Orlic D, Kajstura J, Chimenti S, et al. Bone marrow cells regenerate infarcted myocardium. Nature 2001;410:701-705. [PubMed: 11287958]

8. Wu GD, Nolta JA, Jin YS, et al. Migration of mesenchymal stem cells to heart allografts during chronic rejection. Transplantation 2003;75:679-685. [PubMed: 12640309]

9. Jackson KA, Majka SM, Wang H, et al. Regeneration of ischemic cardiac muscle and vascular endothelium by adult stem cells. J Clin Invest 2001;107:1395-1402. [PubMed: 11390421]

10. Zimmet JM, Hare JM. Emerging role for bone marrow derived mesenchymal stem cells in myocardial regenerative therapy. Basic Res Cardiol 2005;100:471-481. [PubMed: 16237508]

11. Davidoff AM, Ng CY, Brown P, et al. Bone marrow-derived cells contribute to tumor neovasculature and, when modified to express an angiogenesis inhibitor, can restrict tumor growth in mice. Clin Cancer Res 2001;7:2870-2879. [PubMed: 11555605]

12. Crisa L, Cirulli V, Smith KA, et al. Human cord blood progenitors sustain thymic T-cell development and a novel form of angiogenesis. Blood 1999;94:3928-3940. [PubMed: 10572110]

13. Peichev M, Naiyer AJ, Pereira D, et al. Expression of VEGFR-2 and AC133 by circulating human $\mathrm{CD}^{+} 4^{+}$cells identifies a population of functional endothelial precursors. Blood 2000;95:952-958. [PubMed: 10648408]

14. Le Blanc K. Mesenchymal stromal cells: tissue repair and immune modulation. Cytotherapy 2006;8:559-561. [PubMed: 17148032] -Discusses mechanisms by which allogenic mesenchymal stem cells (MSCs) shelter themseleves from the host immune system.

15. Laflamme MA, Murry CE. Regenerating the heart. Nat Biotechnol 2005;23:845-856. [PubMed: 16003373]

16. Chen J, Li Y, Katakowski M, et al. Intravenous bone marrow stromal cell therapy reduces apoptosis and promotes endogenous cell proliferation after stroke in female rat. J Neurosci Res 2003;73:778-786. [PubMed: 12949903]

17. Li Y, Chen J, Zhang CL, et al. Gliosis and brain remodeling after treatment of stroke in rats with marrow stromal cells. Glia 2005;49:407-417. [PubMed: 15540231]

18. Murphy JM, Fink DJ, Hunziker EB, et al. Stem cell therapy in a caprine model of osteoarthritis. Arthritis Rheum 2003;48:3464-3474. [PubMed: 14673997]

19. Rosova I, Dao M, Capoccia B, et al. Hypoxic preconditioning results in increased motility and improved therapeutic potential of human mesenchymal stem cells. Stem Cells 2008;26(8):21732182. [PubMed: 18511601]

20. Kinnaird T, Stabile E, Burnett MS, et al. Marrow-derived stromal cells express genes encoding a broad spectrum of arteriogenic cytokines and promote in vitro and in vivo arteriogenesis through paracrine mechanisms. Circ Res 2004;94:678-685. [PubMed: 14739163]

21. Gnecchi M, He H, Noiseux N, et al. Evidence supporting paracrine hypothesis for Akt-modified mesenchymal stem cell-mediated cardiac protection and functional improvement. FASEB J 2006;20:661-669. [PubMed: 16581974] 
22. Meyerrose T, De Ugarte D, Hofling A, et al. In vivo distribution of human adipose-derived mesenchymal stem cells in novel xenotransplantation models. Stem Cells 2007;25:220-227. [PubMed: 16960135]

23. Meyerrose TE, Roberts M, Ohlemiller KK, et al. Lentiviral-transduced human mesenchymal stem cells persistently express therapeutic levels of enzyme in a xenotransplantation model of human disease. Stem Cells 2008;26:1713-1722. [PubMed: 18436861]

24. Bauer G, Dao MA, Case SS, et al. In vivo biosafety model to assess the risk of adverse events from retroviral and lentiviral vectors. Mol Ther 2008;16:1308-1315. [PubMed: 18461052] - Represents a decade-long biosafety study that confirmed the safety of genetically engineered human MSCs, tested in vivo.

25. Dao MA, Pepper KA, Nolta JA. Long-term cytokine production from engineered primary human stromal cells influences human hematopoiesis in an in vivo xenograft model. Stem Cells 1997;15:443-454. [PubMed: 9402657] - One of the earliest reports of the durability and safety of systemic production of growth factors from genetically engineered human MSCs.

26. Nolta, J. Genetic Engineering of Mesenchymal Stem Cells. Springer; Dordrecht, The Netherlands: 2006.

27. Capoccia BJ, Robson DL, Levac KD, et al. Revascularization of ischemic limbs after transplantation of human bone marrow cells with high aldehyde dehydrogenase activity. Blood 2009;113:5340-5351. [PubMed: 19324906]

28. Danielyan L, Schafer R, von Ameln-Mayerhofer A, et al. Intranasal delivery of cells to the brain. Eur J Cell Biol 2009;88:315-324. [PubMed: 19324456]

29. Crigler L, Robey RC, Asawachaicharn A, et al. Human mesenchymal stem cell subpopulations express a variety of neuroregulatory molecules and promote neuronal cell survival and neuritogenesis. Exp Neurol 2006;198:54-64. [PubMed: 16336965] -'Comprehensive examination of the effects of MSCs on neurons.

30. Munoz JR, Stoutenger BR, Robinson AP, et al. Human stem/progenitor cells from bone marrow promote neurogenesis of endogenous neural stem cells in the hippocampus of mice. Proc Natl Acad Sci USA 2005;102:18171-18176. [PubMed: 16330757]

31. Kassis I, Grigoriadis N, Gowda-Kurkalli B, et al. Neuroprotection and immunomodulation with mesenchymal stem cells in chronic experimental autoimmune encephalomyelitis. Arch Neurol 2008;65:753-761. [PubMed: 18541795] -In vivo MSC-based neuroprotection model.

32. Tolar J, Nauta AJ, Osborn MJ, et al. Sarcoma derived from cultured mesenchymal stem cells. Stem Cells 2007;25(2):371-379. [PubMed: 17038675]

33. Kidd S, Spaeth E, Dembinski JL, et al. Direct evidence of mesenchymal stem cell tropism for tumor and wounding microenvironments using in vivo bioluminescent imaging. Stem Cells 2009;27:2614-2623. [PubMed: 19650040]

34. Kidd S, Spaeth E, Klopp A, et al. The (in) auspicious role of mesenchymal stromal cells in cancer: be it friend or foe. Cytotherapy 2008;10:657-667. [PubMed: 18985472]

35. Kim HJ, McMillan E, Han F, et al. Regionally specified human neural progenitor cells derived from the mesencephalon and forebrain undergo increased neurogenesis following overexpression of ASCL1. Stem Cells 2009;27:390-398. [PubMed: 19008346]

36. Sharp J, Keirstead HS. Stem cell-based cell replacement strategies for the central nervous system. Neurosci Lett 2009;456:107-111. [PubMed: 19429144]

37. Hardy SA, Maltman DJ, Przyborski SA. Mesenchymal stem cells as mediators of neural differentiation. Curr Stem Cell Res Ther 2008;3:43-52. [PubMed: 18220922] --Effects of MSCs on differentiation from neural precursors.

38. Rechavi O, Goldstein I, Kloog Y. Intercellular exchange of proteins: the immune cell habit of sharing. FEBS Lett 2009;583:1792-1799. [PubMed: 19289124]

39. Spees JL, Olson SD, Whitney MJ, et al. Mitochondrial transfer between cells can rescue aerobic respiration. Proc Natl Acad Sci USA 2006;103:1283-1288. [PubMed: 16432190] --An important paradigm-shifting study that definitively demonstrated that human MSCs can transfer components as large as mitochondria to other, damaged cells, through direct cell-to-cell trafficking.

40. Wilkins A, Kemp K, Ginty M, et al. Human bone marrow-derived mesenchymal stem cells secrete brain-derived neurotrophic factor which promotes neuronal survival in vitro. Stem Cell Res. 2009 
Epub ahead of print. -Secretion of brain-derived neurotrophic factor (BDNF) from MSCs promotes neuronal survival.

41. Endo Y, Beauchamp E, Woods D, et al. Wnt-3a and Dickkopf-1 stimulate neurite outgrowth in Ewing tumor cells via a Frizzled3- and c-Jun N-terminal kinase-dependent mechanism. Mol Cell Biol 2008;28:2368-2379. [PubMed: 18212053]

42. Etheridge SL, Spencer GJ, Heath DJ, et al. Expression profiling and functional analysis of wnt signaling mechanisms in mesenchymal stem cells. Stem Cells 2004;22:849-860. [PubMed: 15342948]

43. Gregory CA, Singh H, Perry AS, et al. The Wnt signaling inhibitor dickkopf-1 is required for reentry into the cell cycle of human adult stem cells from bone marrow. J Biol Chem 2003;278:28067-28078. [PubMed: 12740383]

44. Allen J, Chilton JK. The specific targeting of guidance receptors within neurons: who directs the directors? Dev Biol 2009;327:4-11. [PubMed: 19121301]

45. Aizman I, Tate CC, McGrogan M, et al. Extracellular matrix produced by bone marrow stromal cells and by their derivative, SB623 cells, supports neural cell growth. J Neurosci Res 2009;87:3198-3206. [PubMed: 19530164]

46. Croft AP, Przyborski SA. Mesenchymal stem cells expressing neural antigens instruct a neurogenic cell fate on neural stem cells. Exp Neurol 2009;216:329-341. [PubMed: 19159625]

47. Boudreau RL, McBride JL, Martins I, et al. Nonallele-specific silencing of mutant and wild-type huntingtin demonstrates therapeutic efficacy in Huntington's disease mice. Mol Ther 2009;17:1053-1063. [PubMed: 19240687]

48. Harper SQ, Staber PD, He X, et al. RNA interference improves motor and neuropathological abnormalities in a Huntington's disease mouse model. Proc Natl Acad Sci USA 2005;102:5820 5825. [PubMed: 15811941]

49. DiFiglia M, Sena-Esteves M, Chase K, et al. Therapeutic silencing of mutant huntingtin with siRNA attenuates striatal and cortical neuropathology and behavioral deficits. Proc Natl Acad Sci USA 2007;104:17204-17209. [PubMed: 17940007]

50. Wang YL, Liu W, Wada E, et al. Clinicopathological rescue of a model mouse of Huntington's disease by siRNA. Neurosci Res 2005;53:241-249. [PubMed: 16095740]

51. Lombardi MS, Jaspers L, Spronkmans C, et al. A majority of Huntington's disease patients may be treatable by individualized allele-specific RNA interference. Exp Neurol 2009;217:312-319. [PubMed: 19289118]

52. Pfister EL, Kennington L, Straubhaar J, et al. Five siRNAs targeting three SNPs may provide therapy for three-quarters of Huntington's disease patients. Curr Biol 2009;19:774-778. [PubMed: 19361997]

53. Zhang Y, Engelman J, Friedlander RM. Allele-specific silencing of mutant Huntington's disease gene. J Neurochem 2009;108:82-90. [PubMed: 19094060]

54. Lescaudron L, Unni D, Dunbar GL. Autologous adult bone marrow stem cell transplantation in an animal model of Huntington's disease: behavioral and morphological outcomes. Int J Neurosci 2003;113:945-956. [PubMed: 12881187]

55. Bantubungi K, Blum D, Cuvelier L, et al. Stem cell factor and mesenchymal and neural stem cell transplantation in a rat model of Huntington's disease. Mol Cell Neurosci 2008;37:454-470. [PubMed: 18083596]

56. Amin EM, Reza BA, Morteza BR, et al. Microanatomical evidences for potential of mesenchymal stem cells in amelioration of striatal degeneration. Neurol Res 2008;30:1086-1090. [PubMed: 18768110] -MSCs could delay striatal decline in a Huntingdon's disease (HD) model.

57. Watts C, McNamara IR, Dunnett SB. Volume and differentiation of striatal grafts in rats: relationship to the number of cells implanted. Cell Transplant 2000;9:65-72. [PubMed: 10784068]

58. Delcroix GJ, Jacquart M, Lemaire L, et al. Mesenchymal and neural stem cells labeled with HEDP-coated SPIO nanoparticles: in vitro characterization and migration potential in rat brain. Brain Res 2009;1255:18-31. [PubMed: 19103182]

59. Sadan O, Shemesh N, Barzilay R, et al. Migration of neurotrophic factors-secreting mesenchymal stem cells toward a quinolinic acid lesion as viewed by magnetic resonance imaging. Stem Cells 
2008;26:2542-2551. [PubMed: 18635865] --Demonstrates that MSCs migrated specifically to the region of striatal damage after infusion into the brain in an HD rat model.

60. Sadan O, Shemesh N, Cohen Y, et al. Adult neurotrophic factor-secreting stem cells: a potential novel therapy for neurodegenerative diseases. Isr Med Assoc J 2009;11:201-204. [PubMed: 19603590]

61. Bachoud-Levi AC, Gaura V, Brugieres P, et al. Effect of fetal neural transplants in patients with Huntington's disease 6 years after surgery: a long-term follow-up study. Lancet Neurol 2006;5:303-309. [PubMed: 16545746]

62. Gaura V, Bachoud-Levi AC, Ribeiro MJ, et al. Striatal neural grafting improves cortical metabolism in Huntington's disease patients. Brain 2004;127:65-72. [PubMed: 14607797]

63. Canals JM, Pineda JR, Torres-Peraza JF, et al. Brain-derived neurotrophic factor regulates the onset and severity of motor dysfunction associated with enkephalinergic neuronal degeneration in Huntington's disease. J Neurosci 2004;24:7727-7739. [PubMed: 15342740]

64. Her LS, Goldstein LS. Enhanced sensitivity of striatal neurons to axonal transport defects induced by mutant huntingtin. J Neurosci 2008;28:13662-13672. [PubMed: 19074039]

65. Wu LL, Fan Y, Li S, et al. Huntingtin associated protein-1 interacts with proBDNF and mediates its transport and release. J Biol Chem 2009;285(8):5614-5623. [PubMed: 19996106]

66. Gharami K, Xie Y, An JJ, et al. Brain-derived neurotrophic factor over-expression in the forebrain ameliorates Huntington's disease phenotypes in mice. J Neurochem 2008;105:369-379. [PubMed: 18086127]

67. Zuccato C, Cattaneo E. Brain-derived neurotrophic factor in neurodegenerative diseases. Nature Rev 2009;5:311-322.-Highlights the importance of BDNF in ameliorating the neurodegeneration that occurs in HD.

68. Alberch J, Perez-Navarro E, Canals JM. Neurotrophic factors in Huntington's disease. Prog Brain Res 2004;146:195-229. [PubMed: 14699966]

69. Dey ND, Bombard MC, Roland BP, et al. Genetically-engineered mesenchymal stem cells reduce behavioral deficits in the YAC 128 mouse model of Huntington's disease. Behav Brain Res 2010;214(2):193-200. [PubMed: 20493905] - Both BDNF-modified and non-modified MSCs had significant effects in reducing the behavioral defects in an HD mouse model.

70. Nolta JA, Dao MA, Wells S, et al. Transduction of pluripotent human hematopoietic stem cells demonstrated by clonal analysis after engraftment in immune-deficient mice. Proc Natl Acad Sci USA 1996;93:2414-2419. [PubMed: 8637888]

71. Nolta JA, Kohn DB. Comparison of the effects of growth factors on retroviral vector-mediated gene transfer and the proliferative status of human hematopoietic progenitor cells. Hum Gene Ther 1990;1:257-268. [PubMed: 2081193]

72. Tsark E, Dao M, Wang X, et al. IL-7 enhances the responsiveness of human T cells that develop in the bone marrow of athymic mice. J Immunol 2001;166:170-181. [PubMed: 11123290]

73. Wang X, Ge S, McNamara G, et al. Albumin expressing hepatocyte-like cells develop in the livers of immune-deficient mice transmitted with highly purified human hematopoietic stem cells. Blood 2003;101(10):4201-4208. [PubMed: 12560238]

74. Nolta JA, Hanley MB, Kohn DB. Sustained human hematopoiesis in immunodeficient mice by cotransplantation of marrow stroma expressing human interleukin-3: analysis of gene transduction of long-lived progenitors. Blood 1994;83:3041-3051. [PubMed: 7514050]

75. Meyerrose, T.; Rosova, I.; Dao, M., et al. Establishment and Transduction of Primary Human Stromal/ Mesenchymal Stem Cell Monolayers. Kluwer Academic Publishers; Dordrecht, The Netherlands: 2006.

76. Vucic S, Kiernan MC. Pathophysiology of neurodegeneration in familial amyotrophic lateral sclerosis. Curr Mol Med 2009;9:255-272. [PubMed: 19355908]

77. Maxwell MM. RNAi applications in therapy development for neurodegenerative disease. Curr Pharm Des 2009;15:3977-3991. [PubMed: 19751205]

78. Kim SU, de Vellis J. Stem cell-based cell therapy in neurological diseases: a review. J Neurosci Res 2009;87:2183-2200. [PubMed: 19301431]

79. Ionov ID. Survey of ALS-associated factors potentially promoting $\mathrm{Ca}^{2+}$ overload of motor neurons. Amyotroph Lateral Scler 2007;8:260-265. [PubMed: 17917848] 
80. Julien JP, Kriz J. Transgenic mouse models of amyotrophic lateral sclerosis. Biochim Biophys Acta 2006;1762:1013-1024. [PubMed: 16675207]

81. Bruijn LI, Miller TM, Cleveland DW. Unraveling the mechanisms involved in motor neuron degeneration in ALS. Annu Rev Neurosci 2004;27:723-749. [PubMed: 15217349]

82. Zhao CP, Zhang C, Zhou SN, et al. Human mesenchymal stromal cells ameliorate the phenotype of SOD1-G93A ALS mice. Cytotherapy 2007;9:414-426. [PubMed: 17786603] --Demonstrates the positive effects of MSCs in an amyotrophic lateral sclerosis (ALS) model.

83. Vercelli A, Mereuta OM, Garbossa D, et al. Human mesenchymal stem cell transplantation extends survival, improves motor performance and decreases neuroinflammation in mouse model of amyotrophic lateral sclerosis. Neurobiol Dis 2008;31:395-405. [PubMed: 18586098] -Demonstrates the positive effects of MSCs in an in vivo ALS model.

84. Morita E, Watanabe Y, Ishimoto M, et al. A novel cell transplantation protocol and its application to an ALS mouse model. Exp Neurol 2008;213:431-438. [PubMed: 18691571]

85. Fischer LR, Culver DG, Tennant $\mathrm{P}$, et al. Amyotrophic lateral sclerosis is a distal axonopathy: evidence in mice and man. Exp Neurol 2004;185:232-240. [PubMed: 14736504]

86. Klein SM, Behrstock S, McHugh J, et al. GDNF delivery using human neural progenitor cells in a rat model of ALS. Hum Gene Ther 2005;16:509-521. [PubMed: 15871682]

87. Suzuki M, McHugh J, Tork C, et al. GDNF secreting human neural progenitor cells protect dying motor neurons, but not their projection to muscle, in a rat model of familial ALS. PLoS ONE 2007;2:e689. [PubMed: 17668067] --Human GDNF-expressing MSCs had significant effects on ameliorating ALS symptoms.

88. Suzuki M, McHugh J, Tork C, et al. Direct muscle delivery of GDNF with human mesenchymal stem cells improves motor neuron survival and function in a rat model of familial ALS. Mol Ther 2008;16:2002-2010. [PubMed: 18797452]

89. Funakoshi H, Ohya W, Kadoyama K, et al. ALS and neurotrophic factors - HGF as a novel neurotrophic and neuroregenerative factor. Brain Nerve 2007;59:1195-1202. [PubMed: 17969361]

90. Kadoyama K, Funakoshi H, Ohya W, et al. Hepatocyte growth factor (HGF) attenuates gliosis and motoneuronal degeneration in the brainstem motor nuclei of a transgenic mouse model of ALS. Neurosci Res 2007;59:446-456. [PubMed: 17935811]

91. Donsante A, Miller DG, Li Y, et al. AAV vector integration sites in mouse hepatocellular carcinoma. Science 2007;317:477. [PubMed: 17656716]

92. Nagano I, Ilieva H, Shiote M, et al. Therapeutic benefit of intrathecal injection of insulin-like growth factor-1 in a mouse model of amyotrophic lateral sclerosis. J Neurol Sci 2005;235:61-68. [PubMed: 15990113]

93. Nagano I, Shiote M, Murakami T, et al. Beneficial effects of intrathecal IGF-1 administration in patients with amyotrophic lateral sclerosis. Neurosci Res 2005;27:768-772.

94. Mazzini L, Ferrero I, Luparello V, et al. Mesenchymal stem cell transplantation in amyotrophic lateral sclerosis: a Phase I clinical trial. Exp Neurol 2009;223(1):229-237. [PubMed: 19682989] -MSCs were safely infused into the CNS of ten ALS patients in a Phase I clinical trial.

95. Greene P. Cell-based therapies in Parkinson's disease. Curr Neurol Neurosci Rep 2009;9:292-297. [PubMed: 19515281]

96. Jenner P. Functional models of Parkinson's disease: a valuable tool in the development of novel therapies. Ann Neurol 2008;64(Suppl. 2):S16-S29. [PubMed: 19127585]

97. Jin GZ, Cho SJ, Choi EG, et al. Rat mesenchymal stem cells increase tyrosine hydroxylase expression and dopamine content in ventral mesencephalic cells in vitro. Cell Biol Int 2008;32:1433-1438. [PubMed: 18778785]

98. Bouchez G, Sensebe L, Vourc'h P, et al. Partial recovery of dopaminergic pathway after graft of adult mesenchymal stem cells in a rat model of Parkinson's disease. Neurochem Int 2008;52:13321342. [PubMed: 18372079]

99. Sadan O, Bahat-Stromza M, Barhum Y, et al. Protective effects of neurotrophic factor-secreting cells in a 6-OHDA rat model of Parkinson disease. Stem Cells Dev 2009;18:1179-1190. [PubMed: 19243240]

100. Peterson AL, Nutt JG. Treatment of Parkinson's disease with trophic factors. Neurotherapeutics 2008;5:270-280. [PubMed: 18394569] 
101. Manfredsson FP, Okun MS, Mandel RJ. Gene therapy for neurological disorders: challenges and future prospects for the use of growth factors for the treatment of Parkinson's disease. Curr Gene Ther 2009;9:375-388. [PubMed: 19860652]

102. Ramaswamy S, Soderstrom KE, Kordower JH. Trophic factors therapy in Parkinson's disease. Prog Brain Res 2009;175:201-216. [PubMed: 19660658]

103. Li JY, Englund E, Holton JL, et al. Lewy bodies in grafted neurons in subjects with Parkinson's disease suggest host-to-graft disease propagation. Nat Med 2008;14:501-503. [PubMed: 18391963]

104. Mazzini L, Fagioli F, Boccaletti R, et al. Stem cell therapy in amyotrophic lateral sclerosis: a methodological approach in humans. Amyotroph Lateral Scler Other Motor Neuron Disord 2003;4:158-161. [PubMed: 13129802]

105. Karussis D, Kassis I, Kurkalli BG, et al. Immunomodulation and neuroprotection with mesenchymal bone marrow stem cells (MSCs): a proposed treatment for multiple sclerosis and other neuroimmunological/neurodegenerative diseases. J Neurol Sci 2008;265:131-135. [PubMed: 17610906]

106. Mancardi G, Saccardi R. Autologous haematopoietic stem-cell transplantation in multiple sclerosis. Lancet Neurol 2008;7:626-636. [PubMed: 18565456]

107. Saccardi R, Kozak T, Bocelli-Tyndall C, et al. Autologous stem cell transplantation for progressive multiple sclerosis: update of the European group for blood and marrow transplantation autoimmune diseases working party database. Mult Scler 2006;12:814-823. [PubMed: 17263012]

108. Shevchenko YL, Novik AA, Kuznetsov AN, et al. High-dose immunosuppressive therapy with autologous hematopoietic stem cell transplantation as a treatment option in multiple sclerosis. Exp Hematol 2008;36:922-928. [PubMed: 18468768]

109. Atkins H, Freedman M. Immune ablation followed by autologous hematopoietic stem cell transplantation for the treatment of poor prognosis multiple sclerosis. Methods Mol Biol 2009;549:231-246. [PubMed: 19378207]

110. Burt RK, Loh Y, Cohen B, et al. Autologous non-myeloablative haemopoietic stem cell transplantation in relapsing-remitting multiple sclerosis: a Phase I/II study. Lancet Neurol 2009;8:244-253. [PubMed: 19186105]

111. Rogojan C, Frederiksen JL. Hematopoietic stem cell transplantation in multiple sclerosis. Acta Neurol Scand 2009;120:371-382. [PubMed: 19785643]

112. Bonab MM, Yazdanbakhsh S, Lofti J. Does mesenchymal stem cell therapy help multiple sclerosis patients? Report of a pilot study. Iran J Immunol 2007;4:50-57. [PubMed: 17652844]

113. Liang J, Zhang H, Hua B, et al. Allogeneic mesenchymal stem cells transplantation in treatment of multiple sclerosis. Mult Scler 2009;15:644-646. [PubMed: 19389752]

114. Zhang ZX, Guan LX, Zhang K, et al. A combined procedure to deliver autologous mesenchymal stromal cells to patients with traumatic brain injury. Cytotherapy 2008;10:134-139. [PubMed: 18368592]

115. Ting AE, Mays RW, Frey MR, et al. Therapeutic pathways of adult stem cell repair. Crit Rev Oncol Hematol 2008;65:81-93. [PubMed: 18032062]

116. Mays RW, van't Hof W, Ting AE, et al. Development of adult pluripotent stem cell therapies for ischemic injury and disease. Expert Opin Biol Ther 2007;7:173-184. [PubMed: 17250456]

117. Hare JM, Traverse JH, Henry TD, et al. A randomized, double-blind, placebo-controlled, doseescalation study of intravenous adult human mesenchymal stem cells (prochymal) after acute myocardial infarction. J Am Coll Cardiol 2009;54:2277-2286. [PubMed: 19958962]

118. Newman RE, Yoo D, LeRoux MA, et al. Treatment of inflammatory diseases with mesenchymal stem cells. Inflamm Allergy Drug Targets 2009;8:110-123. [PubMed: 19530993]

119. Dryden GW. Overview of stem cell therapy for Crohn's disease. Expert Opin Biol Ther 2009;9:841-847. [PubMed: 19527107] 


\section{Websites}

201. Karussis, D. ClinicalTrials.gov. A service of the US National Institutes of Health; 2010. multiple sclerosis AND stem cell. http://clinicaltrials.gov/ct2/show/NCT00781872

202. ClinicalTrials.gov. A service of the US National Institutes of Health. multiple sclerosis AND stem cell. Jun 1. 2010

http://clinicaltrials.gov/ct2/results?term=mesenchymal+stem+cells+multiple+sclerosis+

203. Clinical Trials http://clinicaltrials.gov

Regen Med. Author manuscript; available in PMC 2011 November 1. 

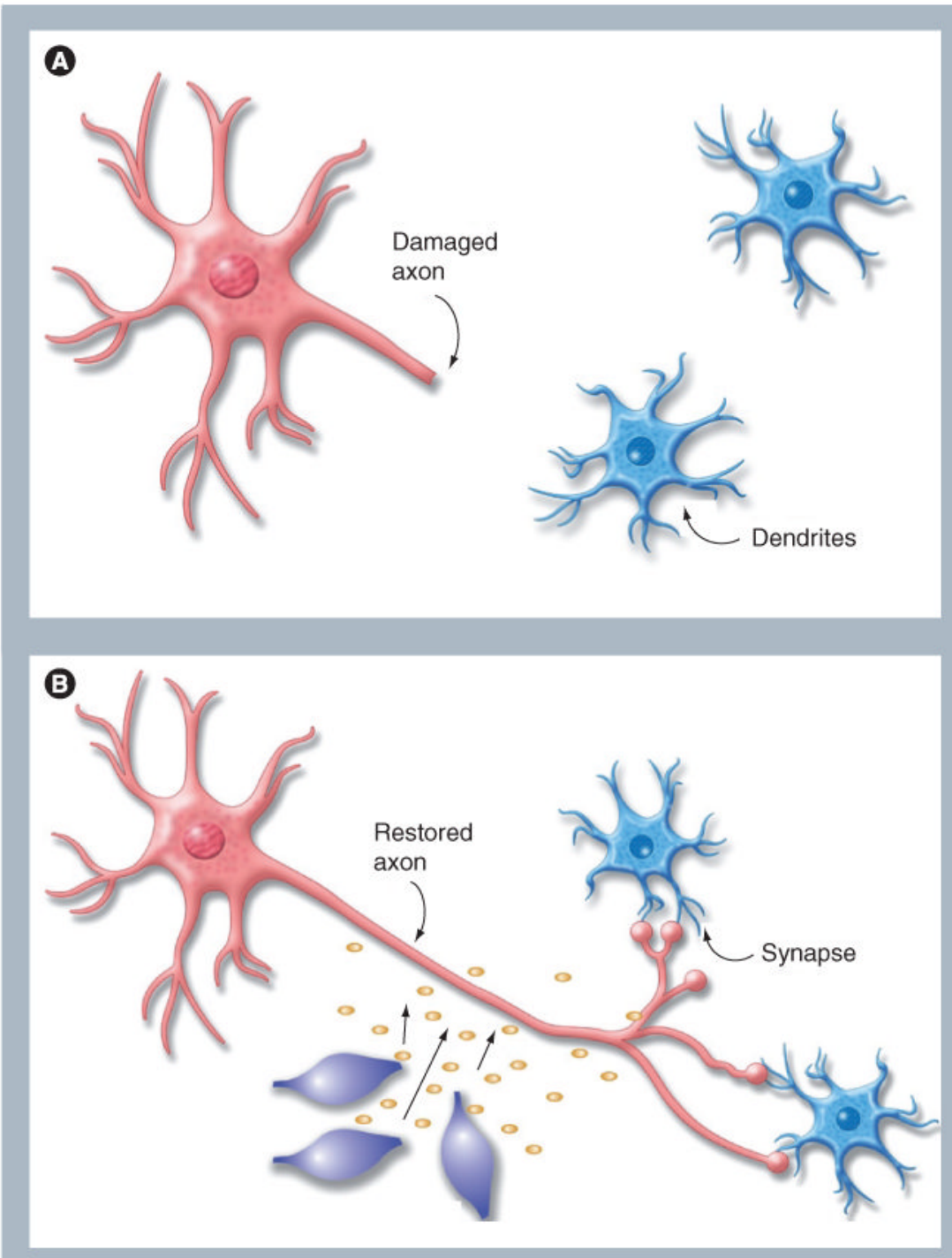

Figure 1. Mesenchymal stem cell-mediated neurotrophic factor secretion to promote neuritogenesis and synaptic connection between damaged neurons

(A) Damaged neurons 'round-up' and retract axons, this prevents effective signaling between cells in the neural network. (B) Mesenchymal stem cells can promote axon extension to restore synaptic connections between neurons by secreting neurotrophic factors. Additional benefits from mesenchymal stem cells in the brain tissue are reduction in inflammation at sites of damage, increased vascularization, reduction of levels of free radicals and a local reduction in apoptosis of damaged cells. 